increasing worldwide. This presentation purposes to report findings from the Korean medical radiation workers' study.

Methods Data on all diagnostic medical radiation workers enrolled at the national dose registry between 1996 and 2011 $(n=94,396)$ were merged with the death and cancer incidence data with coverage through the end of 2015. We reconstructed historical radiation doses and estimated organ-specific doses for all workers. The cancer risks were calculated using standardized mortality ratios (SMRs), standardized incidence ratios (SIRs), excess relative risk (ERR), and lifetime attributable risk (LAR). For radiologic technologists $(n=12,906)$, we conducted a survey and merged the data with the national health insurance data of 2006-2016 for investigation of non-cancer diseases.

Results The mean cumulative badge doses for all workers were $10.6 \mathrm{mSv}$ (men) and $2.7 \mathrm{mSv}$ (women). Medical radiation workers have more favorable mortality than in general population for all causes of death among men $(S M R=0.45)$ and women $(\mathrm{SMR}=0.49)$. Based on 2192 of primary cancer cases, the SIR for all cancers significantly decreased in men $(\mathrm{SIR}=0.88)$ and increased in women $(\mathrm{SIR}=1.10)$. However, there were no significant ERRs of all cancer incidences in both men and women. LARs for all cancer combined ranged from 9 to 402 per 100000 varied by sex and job title. Among radiologic technologists, the odds ratios for cardiovascular diseases showed a significant increasing trend with colon doses (trend $\mathrm{p}=0.024$ ) after adjusting for potential risk factors.

Conclusions Our findings provide some evidence of occupation radiation exposure and its health effects among medical radiation workers. The risks were generally small but not acceptable at high risk groups. Continuous monitoring and further followup is warranted to optimize the work practices for the protection of potential health risks in medical radiation workers.

\section{O6B.7 ARISTOLOCHIC ACID AND THE RISK OF CANCERS IN PATIENTS WITH DIABETES}

${ }^{1}$ Chi-Jen Chen, ${ }^{2}$ Wei-Che Chiu, ${ }^{3}$ Yao-Hsien Tseng, ${ }^{4}$ Yao-Hsu Yang, ${ }^{1}$ Chien-Mu Lin, ${ }^{5} H$ siaoYu Yang, ${ }^{6}$ Pat Doyle, ${ }^{5}$ Pau-Chung Chen. ${ }^{1}$ Taipei Medical University, Taipei, Taiwan; ${ }^{2}$ Cathay General Hospital, Taipei, Taiwan; ${ }^{3}$ Taichung Veterans General Hospital, Taipei, Taiwan; ${ }^{4}$ Chang Gung Memorial Hospital, Chiayi County, Taiwan; ${ }^{5}$ National Taiwan University, Taipei, Taiwan; ' London School of Hygiene and Tropical Medicine, London, UK

\subsection{6/OEM-2019-EPI.148}

Background The purpose of this study was to investigate the association between the use of herbal medicine containing aristolochic acid (AA) and the risk of cancers among patients with diabetes.

Methods We conducted a population-based cohort study on patients older than 18 years who had a diagnosis of diabetes (ICD-9 codes 250) between January 1, 1997 and December 31, 2010. To ensure comparability, we included only patients with diabetes who had visited traditional Chinese medicine clinics between January 1, 1997 and one year before the diagnosis of cancer or the censor dates. The use of herbal medicine containing AA was identified from January 1, 1997 to October 31, 2003 (the ban of herbs containing AA in November 2003). Each patient was individually tracked to identify incident cases of cancer (140-208) between January 1, 1999 and December 31, 2013.

Findings A total of 430377 male and 431956 female patients with diabetes were identified by using the National Health Insurance Research Database in Taiwan. There were 37554 and 31535 cancers during the follow-up period. AA use increased the risks of incident liver (155.0), kidney (189.0), pelvis and ureter (189.1, 189.2), and bladder (188) cancer in male patients with diabetes in a dose-dependent relationship. Similarly, there were increased risks of incident liver, pelvis and ureter, and bladder cancer in female diabetic patients in a dose-dependent manner.

Interpretation Our study suggests that AA exposure plays an important role in the carcinogenesis of liver, kidney, pelvis, ureter, and bladder cancers in patients with diabetes.

\section{Cardiovascular Disease}

\section{C.1 ISCHAEMIC HEART DISEASE AND OCCUPATION: A LINKAGE BETWEEN TWO NEW ZEALAND SURVEYS AND THE INTEGRATED DATA INFRASTRUCTURE}

${ }^{1} \mathrm{M}$ Corbin*, ${ }^{1} \mathrm{~L}$ Barnes, ${ }^{1} \mathrm{HJ}$ Denison, ${ }^{1} \mathrm{~A}$ Eng, ${ }^{1} \mathrm{~A}$ Mannetje, ${ }^{1} \mathrm{D}$ Mclean, ${ }^{1} \mathrm{~L}$ EllisonLoschmann, ${ }^{4} \mathrm{R}$ Jackson, ${ }^{1} \mathrm{~J}$ Douwes. ${ }^{1}$ Centre for Public Health Research, Massey University, Wellington, New Zealand; ${ }^{2}$ Institute of Fundamental Sciences - Statistics, College of Sciences, Massey University, Palmerston North, New Zealand; ${ }^{3}$ Statistical Consulting Unit, The Australian National University, Acton Australian Capital Territory, Australia; ${ }^{4}$ Section of Epidemiology and Biostatistics, School of Population Health, Faculty of Medical and Health Sciences, The University of Auckland, Auckland, New Zealand

\subsection{6/OEM-2019-EPI.149}

Background Associations between ischaemic heart disease (IHD) and occupation are poorly understood. We linked two previously conducted New Zealand workforce surveys with routinely collected health data to assess occupational risk factors of IHD in New Zealand.

Methods Two probability-based sample surveys of the general New Zealand adult population $(2004-2006$; $n=3003)$ and of the New Zealand indigenous peoples (Māori; 2009-2010; $\mathrm{n}=2107$ ), for which occupational history was collected, were linked with health data up to the end of 2017 using Statistics New Zealand individual-level microdata. Incident IHD events were identified using hospitalisations, prescriptions and deaths. The odds ratios associated with ever being employed in occupational groups were estimated by logistic regression adjusting for age, smoking and socio-economic status separately for males and females in each cohort.

Results A total of 282 IHD cases were identified in both surveys. Statistically significant elevated IHD risks were observed for male clerks in the general survey $(\mathrm{OR}=1.60,95 \%$ $\mathrm{CI}=1.02-2.49)$ and agriculture and fishery male workers in the Māori survey $(\mathrm{OR}=1.70,95 \% \mathrm{CI}=1.02-2.82)$. Among females, the odds ratios for agriculture and fishery workers were $1.69(95 \% \mathrm{CI}=0.81-3.51)$ and $1.49(95 \% \mathrm{CI}=0.81-2.75)$ in the general survey and the Māori survey, respectively. A statistically significant increased risk was observed for female plant and machine operators and assemblers in the Māori survey $(\mathrm{OR}=1.87,95 \% \mathrm{CI}=1.05-3.31)$. In the general survey, male plant and machine operators and assemblers had an odds ratio of $1.26(95 \% \mathrm{CI}=0.81-1.95)$. We also identified borderline increased odds ratios for trades workers among males in the general survey $(\mathrm{OR}=1.39,95 \% \mathrm{CI}=0.92-2.12$, $\mathrm{p}$-value $=0.12$ ) and among females in the Māori survey $(\mathrm{OR}=2.26,95 \% \mathrm{CI}=0.98-5.21$, p-value $=0.06)$.

Conclusion This study identified associations between several occupational groups and IHD in the New Zealand population. Further analyses will be conducted to assess specific occupational exposures associated with IHD risk. 